\title{
Evasion differential game of two evaders and one pursuer with integral constraints
}

\begin{abstract}
A simple motion evasion differential game of two evaders and one pursuer was studied. Control functions of all players are subjected to integral constraints. We say that evasion is possible if the state of at least one of the evaders does not coincide with that of the pursuer. We proved that if the total energy of the evaders is greater than or equal to the energy of the pursuer, then evasion is possible. Though the game is considered in a plane, the results of the paper can be easily extended to $\mathbb{R} n$.
\end{abstract}

Keyword: Control; Differential game; Evasion; Integral constraint; Strategy 